\title{
Analyse typologique de la diversité et des performances de l'élevage oasien
}

\author{
Mohamed Taher Sraïri*, Fatima Azahra M'ghar, Mohammed Benidir et Mohammed Bengoumi \\ Département des productions et biotechnologies animales, institut agronomique et vétérinaire Hassan II, BP 6202, Rabat, 10101, Madinate \\ Al Irfane, Maroc
}

\begin{abstract}
Résumé - L'élevage constitue une composante indissociable des systèmes agraires oasiens. Par ses revenus complémentaires, les restitutions de matière organique aux sols et la fourniture d'une force de travail, il contribue à la sécurité alimentaire et à la résilience de ces systèmes. Toutefois, ces milieux arides ont subi récemment des changements profonds, notamment l'augmentation de la pression sur les ressources en sols et eau du fait de l'accroissement démographique, qui ont impacté l'élevage. En outre, l'aspiration des populations oasiennes à de meilleures rémunérations a amplifié la problématique du travail d'astreinte nécessaire à la conduite des troupeaux. La présente étude vise à actualiser la connaissance des systèmes d'élevage des oasis, à travers une analyse typologique de leur structure, de leur conduite et de leurs performances zootechniques. Pour ce faire, 100 exploitations agricoles de la vallée du fleuve Drâa (Centre-Est du Maroc) ont été étudiées. Des analyses statistiques multidimensionnelles ont permis d'identifier quatre types d'élevages distincts, selon la composition des troupeaux, leur conduite alimentaire et les produits élaborés, définis comme suit : « élevage plurispécifique », "élevage basé sur l'espèce bovine », "élevage avec intensification de la production ovine » et « élevage à finalité pastorale ». Ces quatre types ont ensuite été caractérisés par une analyse plus poussée d'individus représentatifs. Les résultats confirment la diversité des options mises en œuvre pour assurer la viabilité de l'élevage et des exploitations agricoles qui l'abritent. La variabilité des stratégies des acteurs implique d'adapter les interventions possibles selon chaque type d'élevage. Cela permettrait de juger les effets des politiques publiques qui ont été testées et de canaliser les efforts du développement agricole et d'orienter les mesures d'appui vers la levée des contraintes les plus handicapantes.
\end{abstract}

Mots clés : incertitude climatique / Maroc / oasis / systèmes d'élevage / typologie

\begin{abstract}
A typological analysis of livestock diversity and performances in the oases. Livestock is a crucial component in the oasis agricultural systems. Given its complementary incomes, the manure for soil fertilization and its draft power, livestock contributes to food security and ensures resilience of these systems. However, oases have recently witnessed significant changes, due to a growing pressure on soil and water resources induced by a rapid demographic expansion. The present study therefore aims at characterizing the different kinds of livestock production systems adopted in the oases, through the establishment of a typology of farms, based on herds' structural parameters as well as their performances. This was achieved in the Drâa River Valley (Center-East of Morocco), where one hundred farms were surveyed. Multivariate statistical analyses have allowed identifying four different types of livestock systems, with regard to herd composition, feed resources used, as well as animal products' outputs. These systems were defined as follows: multispecific livestock rearing, cattle based livestock activities, sheep intensification and rangeland systems. These four distinct types have been further characterized by an additional analysis of representative individuals. Results reveal the diversity of the options adopted by farmers to ensure the viability of their activities, given the assets they manage, in order to address the constraints of the harsh environment. Limited performances imply that possible interventions may be adapted to each type of livestock system. This would allow better assessing the public policies that have been implemented, targeting the efforts of the agricultural development agencies in the area, and orienting public support policies towards the alleviation of the most prominent constraints.
\end{abstract}

Keywords: climate uncertainty / Morocco / oasis / livestock systems / typology

\footnotetext{
* Auteur de correspondance : msrairi@hotmail.com
} 


\section{Introduction}

L'élevage est une composante essentielle des systèmes de production agricole dans les oasis. Il permet d'entretenir la fertilité des sols à travers les épandages des déjections des animaux. Il assure aussi la diversification des sources de revenus des exploitations familiales en valorisant les fourrages et les coproduits des cultures, par les ventes de lait et de viande. L'association « cultures-élevage », dont les complémentarités ont été avancées pour contribuer à expliquer la résilience des exploitations agricoles dans d'autres contextes (Ryschawy et al., 2013), s'avère cruciale pour le maintien de l'écosystème anthropique oasien. En effet, ces régions se définissent comme des espaces cultivés intensivement dans un milieu désertique ou fortement marqué par l'aridité (Jouve, 2012). De ce fait, le bilan hydrique y est largement déficitaire. Sous le palmier dattier, espèce dominante du système oasien, se trouvent installées plusieurs strates de végétation; l'ensemble procure un agroécosystème favorable aux activités culturales. Au Maroc, les oasis se situent dans des zones arides (moins de $150 \mathrm{~mm}$ par an). Environ 1,7 millions de personnes y vivent, soit près de $5 \%$ de la population du pays. Toutefois, ces zones se caractérisent par une vulnérabilité sociale marquée, qui devrait être exacerbée à l'avenir par les effets du changement climatique (Schilling et al., 2012). Ainsi, du fait des revenus limités des activités agricoles de ces régions, et face aux aspirations de mieux-être de leur population, l'émigration est fréquente (de Haas, 2006). Par les revenus qu'elle procure, elle permet des changements perceptibles dans les systèmes agricoles, comme l'introduction de nouvelles spéculations à haut niveau d'intrants, telles que le maraîchage ou l'horticulture (Rignall, 2015). Par ailleurs, les rares références sur l'agriculture et l'élevage oasiens sont le plus souvent issues de travaux de recherche conduits en station (Bouix et al., 1977) et ne sont pas actualisées. Pour l'élevage, l'essentiel des études est centré sur la race ovine D'man, réputée pour sa prolificité (Dollé et al., 1989), mais ne considère pas les autres espèces (bovins, caprins et dromadaires). De plus, ces travaux ne vont pas jusqu'à renseigner les effets des politiques publiques récentes (subventions à la sélection de la race D'man et installation de l'élevage bovin laitier) et les interactions entre le cheptel et la valorisation des ressources alimentaires locales ainsi que les stratégies adoptées par les exploitations agricoles. Aussi, pour pallier ce manque de références, le présent article vise-t-il à établir une typologie des élevages, et à en préciser le fonctionnement, au vu des contraintes qui s'imposent aux productions animales dans le milieu oasien.

\section{Matériel et méthodes}

La zone étudiée est la vallée du Drâa. La surface agricole utile y est de 26000 ha, répartie dans un chapelet d'oasis s'étendant sur près de $200 \mathrm{~km}$, le long du fleuve (Fig. 1). Le climat se caractérise par des précipitations moyennes annuelles inférieures à $110 \mathrm{~mm}$ et des températures estivales élevées, dépassant $48^{\circ} \mathrm{C}$ à l'ombre (Fig. 2). Le long de la vallée un gradient de disponibilité en eau est noté, avec l'apparition de problèmes de salinité et de manque d'eau d'irrigation à l'aval (Karmaoui et al., 2014). De ce fait, l'irrigation à partir des eaux souterraines est généralisée, surtout pour des cultures exigeantes comme les palmiers dattiers et la luzerne.
Cent exploitations détenant des animaux d'élevage ont été étudiées d'avril à août 2015. Elles se répartissaient à raison de 11 à 16 exploitations dans chacune des sept palmeraies localisées le long des oueds Drâa et Maïder. Elles ont été retenues de manière à refléter la diversité des situations agraires et d'élevage, aussi bien en termes d'accès aux sources en eau (de l'amont à l'aval de la vallée) qu'en termes d'espèces animales présentes (part des bovins, camelins, caprins et ovins dans le cheptel). Les entretiens ont été centrés autour des caractéristiques structurelles de l'exploitation (surface totale et agricole utile), le parcellaire et l'assolement, les équipements ainsi que le groupe humain y évoluant. La structure du troupeau a été observée en renseignant les contributions des différentes espèces (bovins, ovins, caprins et camélidés) et les ateliers le constituant (femelles reproductrices, géniteurs, jeunes, etc.). Les mouvements (ventes, achats, naissances et mortalités) des animaux ont été reconstitués sur l'année écoulée. Les rations alimentaires journalières par atelier ont été calculées à partir de celles du jour de l'enquête. Des indicateurs de structure des troupeaux ont été calculés (part de chaque espèce, exprimée en unités gros bétail [UGB]). Les revenus des ventes animales et du lait ainsi que des subventions perçues par UGB ont été déterminés. En outre, l'autonomie fourragère, définie comme la part de l'énergie issue des aliments produits dans l'exploitation par rapport à l'énergie totale de la ration ingérée, a été calculée. Les données obtenues ont fait l'objet d'analyses statistiques descriptives préliminaires, suivies d'analyses multidimensionnelles pour l'établissement de la typologie des élevages.

\section{Résultats}

La majorité des 100 exploitations se caractérisent par une surface exiguë moyenne de 3,2 $\pm 3,9$ ha $\left(1^{\mathrm{er}}\right.$ quartile $=0,92$; médiane $=2,00: 3^{\mathrm{e}}$ quartile $\left.=3,84\right)$. Vingt-quatre exploitations n'ont pas de puits et elles utilisent ceux des voisins en payant le prix de l'exhaure de l'eau. De fait, toutes les exploitations sont irriguées. Les cultures les plus pratiquées sont le blé (38\% de la surface), la luzerne (23\%), l'orge (13\%), la pastèque (13\%); les $13 \%$ restant sont utilisés pour le maraîchage, le maïs, le henné, le melon et les arbres fruitiers (Fig. 3). Le palmier dattier est aussi un des éléments principaux des exploitations ; 85 en ont planté, mais avec des effectifs variables (en moyenne $256 \pm 721$ ), et des extrêmes de 5 à 6000 pieds. Seules neuf exploitations ont recours à de la main-d'œuvre extrafamiliale, mais la contrainte travail demeure prégnante, car les éleveurs s'accordent sur l'astreinte générée par les soins à leurs animaux. La contribution des femmes est remarquable, puisqu'elles participent aux tâches agricoles dans 86 exploitations et constituent l'unique force de travail dans 31 .

Le cheptel, converti en UGB, se compose ainsi : ovins (34\%), camelins (30\%), bovins $(29 \%)$ et caprins $(7 \%)$. Les troupeaux ovins sont surtout de race D'man, mais 13 exploitations ont recours à des croisements avec les races Sardi ou Lacaune. Pour les bovins, le type croisé (lait $\times$ locale) est dominant, même si deux exploitations possèdent uniquement des races importées (Holstein ou Montbéliarde).

Un bilan des apports énergétiques (en unité fourragère lait [UFL]), établi dans les 100 exploitations, démontre que le pâturage représente $26 \%$ des apports énergétiques totaux, grâce aux précipitations relativement abondantes de l'automne 


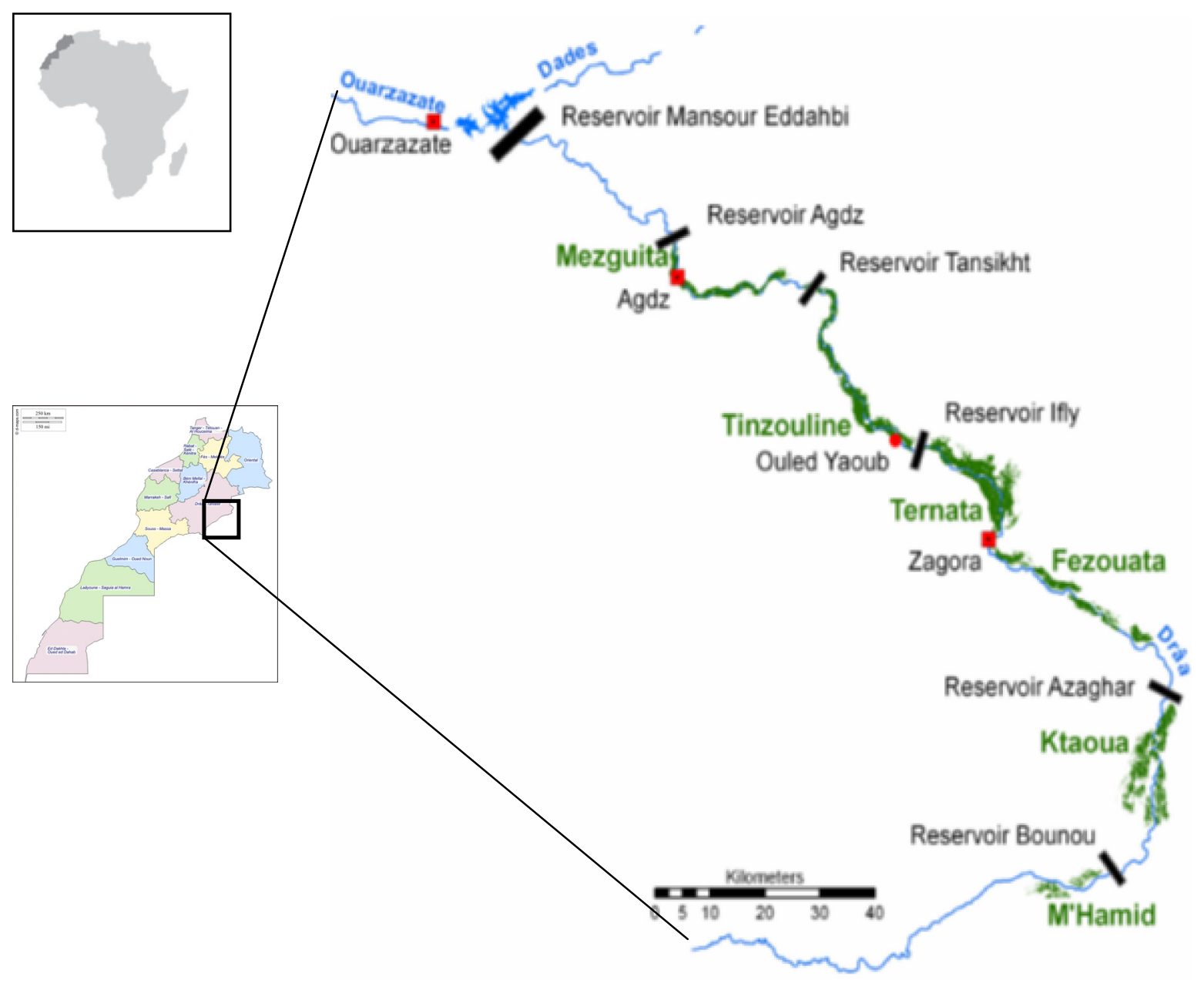

Fig. 1. Localisation géographique de la région d'étude.

Fig. 1. Localization of the study sites in Morocco.

2014. La luzerne contribue à $34 \%$ des apports totaux, sous forme verte ou en foin. Suivent les aliments composés $(9,6 \%)$, les pailles de céréales $(8 \%)$ et les déchets de datte $(5 \%)$ (Fig. 4). Une autre caractéristique de l'élevage et de l'agriculture oasiens est la fréquente adhésion des exploitations à un organisme professionnel, notamment les groupements de l'Association nationale ovine et caprine (ANOC), la coopérative de transformation du lait bovin, ou les coopératives de commercialisation des dattes ou du henné.

$\mathrm{Au}$ vu de la diversité marquée des caractéristiques structurelles et de fonctionnement des troupeaux, une analyse multidimensionnelle s'est imposée. Huit variables quantitatives, peu corrélées entre elles, et illustrant la diversité des exploitations, ont été considérées pour une analyse en composantes principales (ACP). Elles visaient à cerner précisément la réalité des choix des exploitations (importance de l'élevage illustrée par la part des fourrages dans l'assolement total) et dans la structure du cheptel (part des espèces), ainsi que l'origine des aliments (endogènes ou achetés) :

- ressources alimentaires endogènes des exploitations (ressources endogènes [RE]) ;

- apports énergétiques issus des déchets de dattes (AEDD) par rapport à l'énergie totale ingérée ;
- apports énergétiques de la luzerne par rapport à l'énergie totale ingérée (UFLT) ;

- surface des fourrages par rapport à la surface totale (SFST) ;

- apports totaux en UFL par UGB (AETU) ;

- UGB bovines sur UGB totales (UGBT) ;

- UGB caprines sur UGB totales (UGCT) ;

- ventes annuelles par UGB (VAUGB).

Les deux premiers axes factoriels restituent $47,9 \%$ de la variance totale. Ce plan principal a permis une bonne discrimination graphique (Fig. 5) : en abscisse, les ressources alimentaires de l'exploitation (liées aux variables « RE », «SFST », «AEDD») et, en ordonnée, l'élevage bovin (corrélé aux variables «UGBT » et «AETU »). Finalement, quatre types d'élevage ont été identifiés :

- « élevage plurispécifique » $(n=13)$;

- « élevage basé sur l'espèce bovine » $(n=29)$;

- « élevage avec intensification de la production ovine » $(n=39)$;

- « élevage à finalité pastorale » $(n=19)$.

Cette typologie a été présentée aux responsables locaux de l'agriculture qui l'ont validée (Tab. 1). 


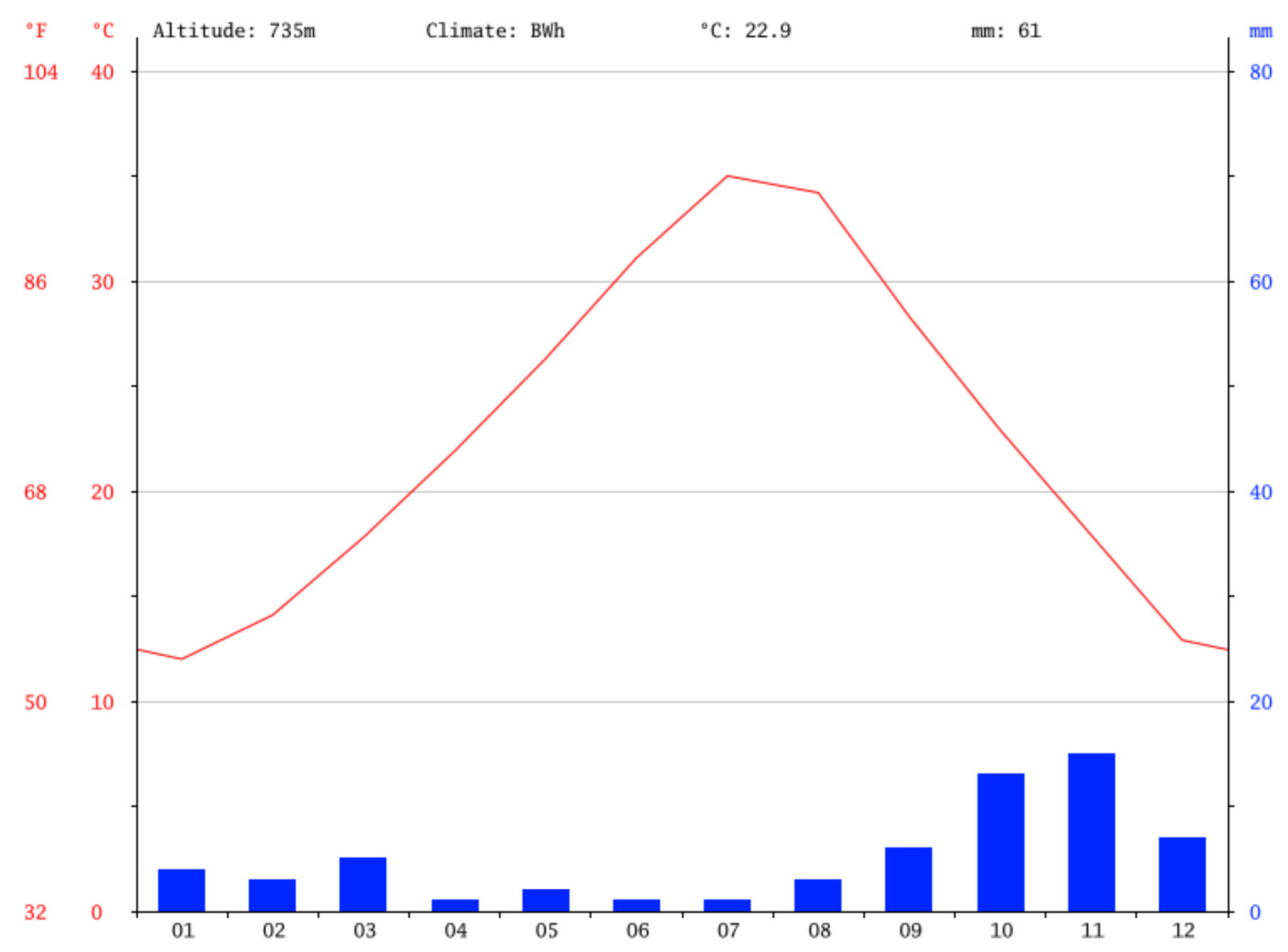

Fig. 2. Diagramme ombrothermique de la ville de Zagora (vallée du Drâa).

Fig. 2. Climograph of Zagora city (Drâa River Valley).

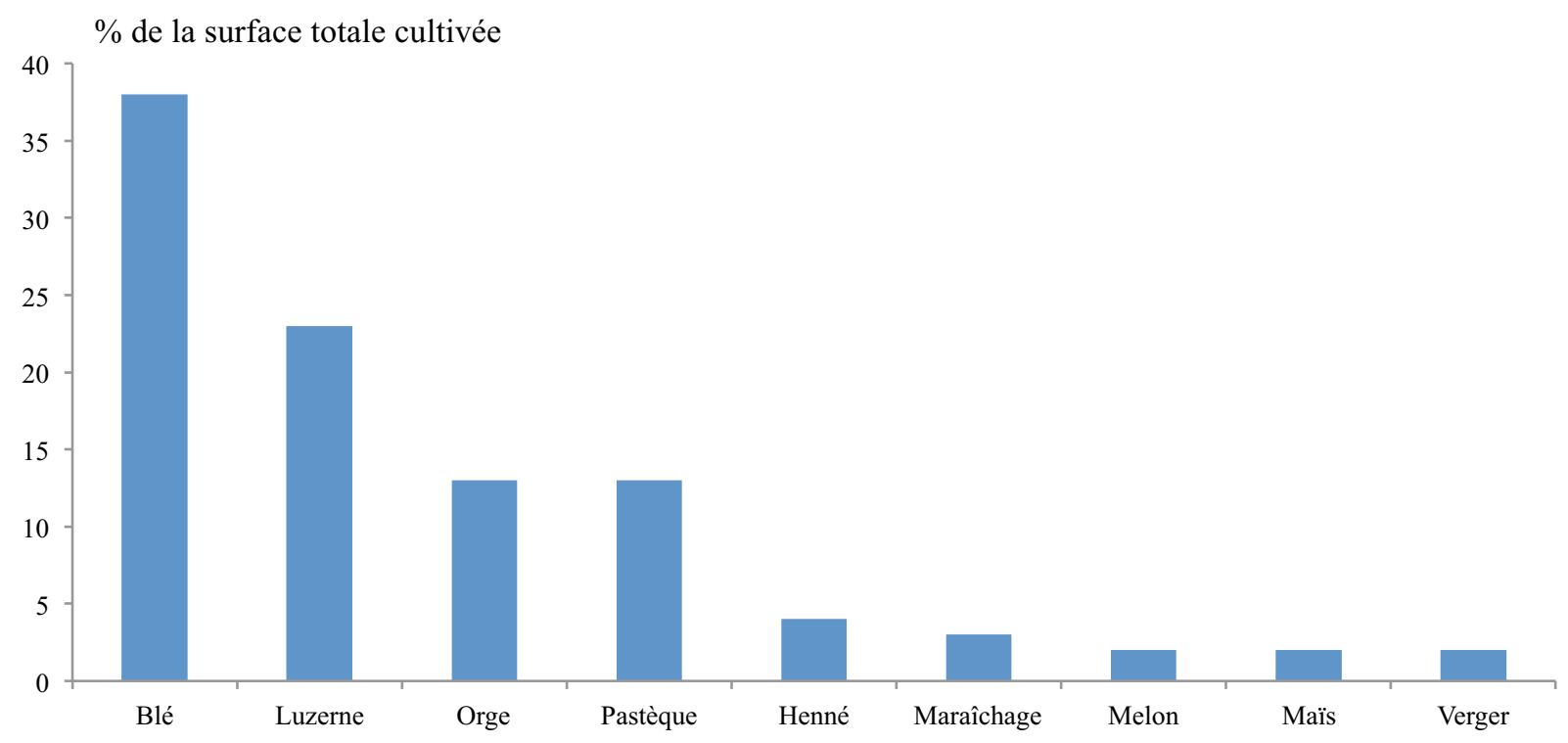

Fig. 3. Répartition des surfaces selon les cultures emblavées dans 100 exploitations du Drâa.

Fig. 3. Allocation of agricultural land to different crops in 100 Drâa farms.

Sur la base de ces quatre types d'élevage identifiés, un approfondissement de l'analyse de leur fonctionnement a été entrepris. Pour chaque type d'élevage, un cas a été décrit. $\mathrm{Ce}$ dernier n'est pas le résultat d'une analyse statistique fondée sur des moyennes, mais la recherche d'une explication cohérente d'un objet complexe - le système d'élevage - autour d'une représentation se rapportant à un cas concret (Tab. 2).

Une exploitation de 1,21 ha, cultivée en luzerne, céréales, cultures maraîchères et henné, illustre le type 1 «élevage plurispécifique »; elle compte 110 palmiers dattiers plantés et 


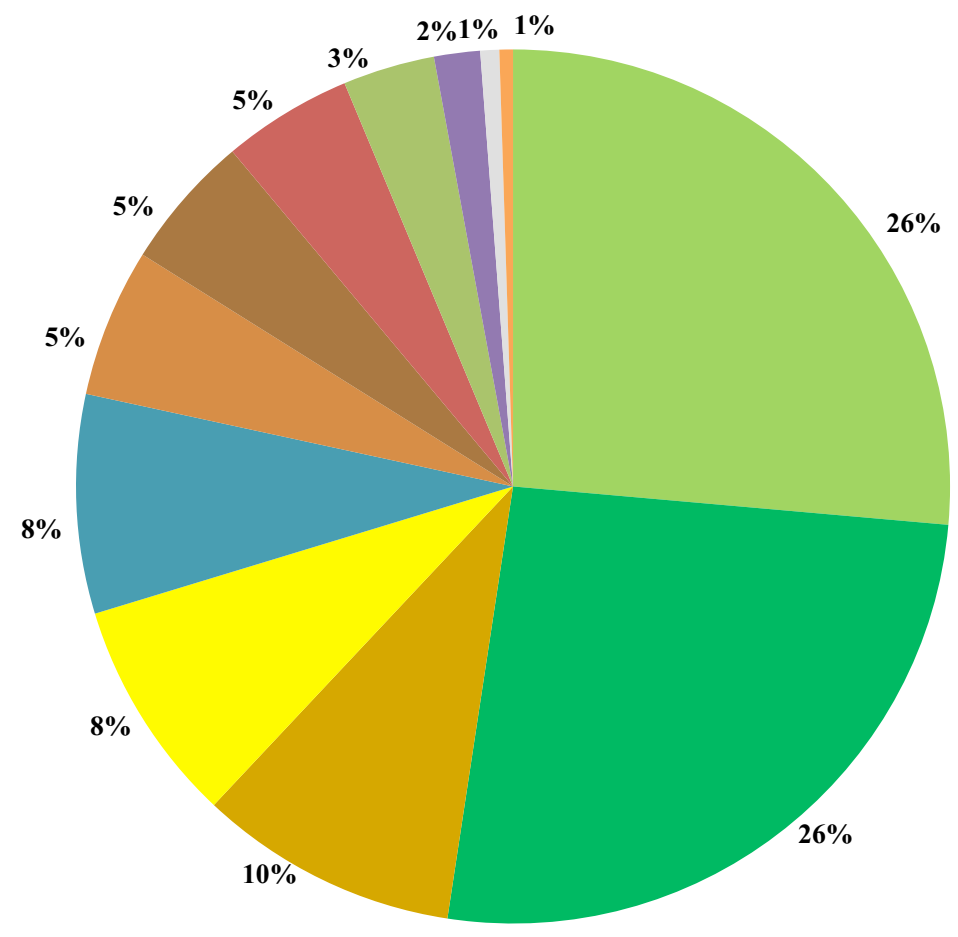

Parcours
Foin de luzerne
Son de blé

$\square$ Luzerne en vert
Grains d'orge
$\square$ Herbes diverses

Aliments composés

- Déchets de dattes

Pain sec

\author{
Paille de céréales \\ - Pulpes sèches de betterave \\ Maïs en vert
}

Fig. 4. Contribution des différentes matières premières aux apports énergétiques totaux.

Fig. 4. Contribution of different resources to total forage ration energy.

un troupeau constitué de deux bovins, dix ovins et cinq caprins. L'effectif bovin a été diminué en 2015 suite à des problèmes d'infécondité. Un volume de 101 de lait est produit quotidiennement, autoconsommé ou en partie offert aux voisins. Le troupeau ovin est au centre des préoccupations de la propriétaire de l'exploitation, qui a bénéficié d'un don de trois brebis D'man de la part d'une institution caritative. La fertilité ovine est de $100 \%$, et la prolificité atteint $175 \%$, tandis que le taux de mortalité est largement supérieur aux normes $(28,5 \%)$. L'alimentation du cheptel repose à près de $100 \%$ sur des RE (luzerne, déchets de datte et paille de blé). Seuls les jeunes en phase de finition reçoivent un complément à base de son et de pulpes sèches de betterave. La main-d'œuvre est aussi exclusivement endogène : la responsable de l'élevage, aidée de son frère et de son père qui assurent l'affouragement et l'abreuvement des animaux. Les sources de revenu ne se limitent pas aux seules ventes de produits agricoles mais comprennent aussi le revenu généré à partir d'une épicerie.

Une exploitation de 6,6 ha a été choisie pour décrire le type 2 , « élevage basé sur l'espèce bovine ». Le blé dur et la luzerne y occupent chacun 3 ha, suivis du henné $(0,5$ ha) et du maraîchage $(0,1$ ha $) ; 300$ pieds de palmiers dattiers ont été récemment plantés. L'exploitation dispose de huit bovins de type croisé (lait $\times$ locale) et 26 ovins. Les bovins constituent $85 \%$ des UGB totales. Un intervalle entre vêlages de 13 mois est enregistré, ainsi qu'une durée de tarissement de trois mois. La production laitière est faible, eu égard au potentiel des vaches de type croisé : 51 par jour par vache en lactation.
Le lait est vendu à la coopérative locale de transformation. L'élevage ovin se caractérise par des performances proches des normes pour la race D'man : fertilité de $100 \%$, prolificité de $200 \%$ et mortinatalité de 8,3\%. L'alimentation du cheptel est en grande partie basée sur les ressources de l'exploitation (84\% de l'apport énergétique total) avec l'addition d'aliments concentrés, sous forme d'orge subventionnée qui a été distribuée aux éleveurs au printemps 2014. Le travail est assuré exclusivement par de la main-d'œuvre familiale. Les sources de revenus sont diverses et comprennent, outre les ventes du henné, des dattes et du croît animal, les versements des membres de la famille ayant une activité extra-agricole.

Le type 3, « élevage avec intensification de la production ovine », est représenté par un cheptel constitué de trois bovins et 31 ovins. Ces animaux évoluent dans une exploitation de 7 ha, cultivée en luzerne ( 1 ha), céréales $(3,4$ ha $)$, pastèque ( 2 ha) et maraîchage (0,6 ha). Quatre cents pieds de palmier dattier sont dénombrés, en addition à un verger qui contient diverses espèces fruitières. Le cheptel bovin se justifie surtout par sa production de lait, nécessaire pour assurer la viabilité des agneaux en cas de portées multiples. Le troupeau ovin destiné à la sélection de la race D'man est stratégique à plusieurs égards : part dans les revenus totaux, mobilisation des ressources alimentaires et temps de travail. Il s'agit de 18 brebis de race D'man, caractérisées par une fertilité de $95 \%$, une prolificité acceptable $(186 \%)$ et un taux de mortinatalité supérieur aux normes $(13,5 \%)$. L'adhésion de l'exploitation à l'ANOC lui a permis de bénéficier d'une subvention de l'ordre de $16000 \mathrm{DH}$ 


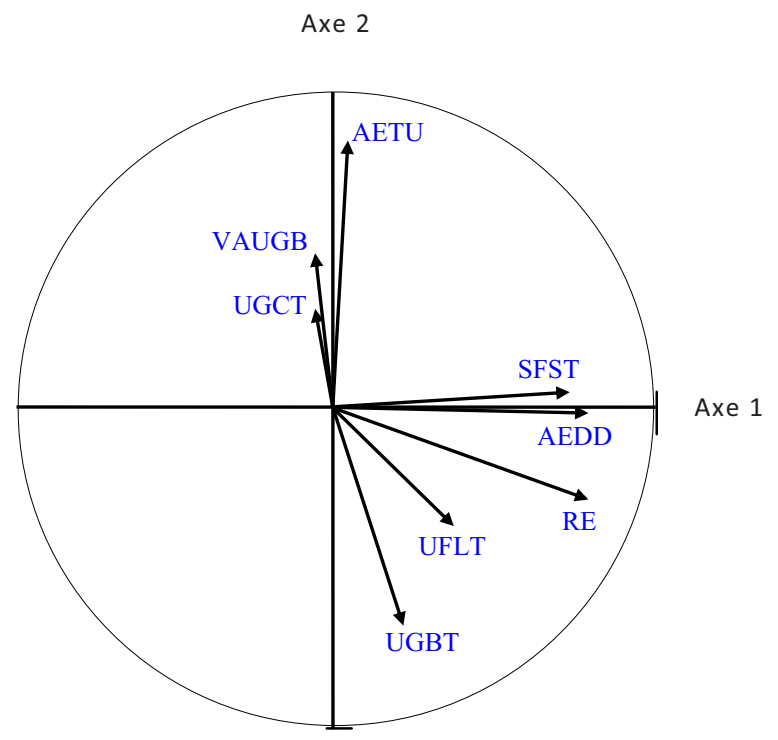

RE : Ressources endogènes ; AEDD : Apports énergétiques issus des déchets de dattes ; SFST : surface des fourrages par rapport à la surface totale ; AETU : apports énergétiques totaux par UGB ; UGBT : Unités gros bétail bovin/UGB totales ; VAUGB : Ventes animales annuelles par UGB ; UGCT : Unités gros bétail caprin/UGB totales ; UFLT : Unités fourragères de la luzerne/UFL totales

Fig. 5. Projection des variables caractérisant les élevages oasiens sur le plan factoriel (axes 1 et 2).

Fig. 5. Projection of variables characterizing oasis livestock systems on factorial plane 1-2.

Tableau 1. Comparaison des types d'élevage identifiés par un test d'analyse de la variance.

Table 1. Comparison of the different livestock system types using an analysis of variance test.

\begin{tabular}{|c|c|c|c|c|}
\hline & Type $1(n=13)$ & Type $2(n=29)$ & Type $3(n=39)$ & Type $4(n=19)$ \\
\hline Effectif moyen des camelins (UGB) & $0,0^{\mathrm{a}}$ & $0,0^{\mathrm{a}}$ & $0,0^{\mathrm{a}}$ & $8,3^{\mathrm{b}}$ \\
\hline UGB bovines/UGB totales (\%) & $19,0^{\mathrm{a}}$ & $70,0^{\mathrm{b}}$ & $13,3^{\mathrm{a}}$ & $0,0^{\mathrm{a}}$ \\
\hline Apports en UFL/UGB totales & $6,8^{\mathrm{a}}$ & $5,4^{\mathrm{b}}$ & $7,6^{\mathrm{a}}$ & $6,6^{\mathrm{a}}$ \\
\hline UFL luzerne/UFL totales $(\%)$ & $52,0^{\mathrm{a}}$ & $61,0^{\mathrm{a}}$ & $49,0^{\mathrm{a}}$ & $21,2^{\mathrm{b}}$ \\
\hline Ventes animales (DH/UGB/an) & $2588^{\mathrm{a}}$ & $2940^{\mathrm{a}}$ & $6567^{\mathrm{b}}$ & $1976^{\mathrm{a}}$ \\
\hline Adhésion à l'ANOC (\%) & $8,0^{\mathrm{a}}$ & $3,0^{\mathrm{a}}$ & $56,0^{\mathrm{b}}$ & $0,0^{\mathrm{a}}$ \\
\hline
\end{tabular}

ANOC : Association nationale ovine et caprine ; UFL : unité fourragère lait ; UGB : unité gros bétail. Les moyennes affectées d'une même lettre sur une même ligne ne sont pas significativement différentes $(p>0,005)$.

(1 450 euros) par an, du fait de la sélection de 32 antenais qui y sont nés. Les RE de l'exploitation n'assurent que $56 \%$ des apports énergétiques totaux, en raison de l'usage d'aliments concentrés achetés, vulgarisés par les techniciens de l'organisation professionnelle ovine et caprine (ANOC). Les travaux agricoles quotidiens sont réalisés par les membres de la famille. Toutefois, des femmes du voisinage aident à la coupe et au transport du fourrage contre une partie de cette récolte. Les dattes, la pastèque, les amandes, le miel et le croît ovin, ainsi que les subventions étatiques, sont les uniques sources de revenu, vu l'absence d'activités extra-agricoles. Les ventes d'animaux ont assuré une somme de $34500 \mathrm{DH}$ (3 140 euros), dont
$28000 \mathrm{DH}$ (2 550 euros) issus des ventes d'agneaux à l'occasion de l'Aïd el Adha.

Le type 4, « élevage à finalité pastorale », est représenté par une exploitation d'un hectare, cultivant 120 palmiers dattiers, de la luzerne ( 0,1 ha) ainsi que des céréales $(0,4$ ha). Le troupeau est constitué de 17 chamelles. Elles sont conduites sur les parcours, relativement riches suite aux précipitations de l'automne 2014. Elles sont libérées dans les parcours sans gardien, mais sont distinguées par un système de marquage grâce auquel chaque éleveur reconnaît son troupeau. En période de disette, l'exploitant renforce l'alimentation du troupeau par de l'orge et des déchets de 
Tableau 2. Analyse comparative des cas-types illustrant chacun des types d'élevage distingués.

Table 2. Comparative analysis of typical examples of each livestock system type.

\begin{tabular}{|c|c|c|c|c|}
\hline & $\begin{array}{l}\text { Élevage plurispécifique } \\
\text { des oasis }\end{array}$ & $\begin{array}{l}\text { Élevage basé } \\
\text { sur l'espèce bovine }\end{array}$ & $\begin{array}{l}\text { Élevage avec } \\
\text { intensification ovine }\end{array}$ & $\begin{array}{l}\text { Élevage à finalité } \\
\text { pastorale }\end{array}$ \\
\hline Surface totale (ha) & 1,21 & 6,6 & 7,0 & 1 \\
\hline Nombre de palmiers dattiers & 110 & 300 & 400 & 120 \\
\hline Nombre de puits & 1 & 3 & 2 & - \\
\hline UGB caprines/UGB totales $(\%)$ & 13 & 0 & 0 & 0 \\
\hline Besoins en UFL/UGB & 6,63 & 6,62 & 7,61 & 4,46 \\
\hline Luzerne/énergie totale $(\%)$ & 62 & 67 & 44 & 0 \\
\hline Déchets de dattes/énergie totale $(\%)$ & 16 & 9 & 0 & 0 \\
\hline Ressources endogènes $(\%)$ & 100 & 84,1 & 56 & 0 \\
\hline Ventes d'animaux (DH/an) & 7000 & 28000 & 44500 & 44000 \\
\hline Subventions (DH/UGB) & 0,0 & 0,0 & 16000 & 0,0 \\
\hline
\end{tabular}

ANOC : Association nationale ovine et caprine ; UFL : unité fourragère lait ; UGB : unité gros bétail.

dattes. La terre est confiée en faire-valoir indirect à un associé qui prélève le quart de la récolte des dattes, et la moitié des grains de céréales. Il bénéficie de la totalité de la luzerne pour son propre troupeau : deux brebis et quatre chèvres en stabulation. Les revenus dépendent donc surtout des activités extra-agricoles des fils de l'exploitant, complétées par les ventes des chamelons : quatre sont sevrés en moyenne par an et vendus à un prix moyen individuel de 12000 DH (1 100 euros).

L'analyse révèle aussi que la situation géographique des différents types dépend des spécificités locales. Ainsi, le type 1 est retrouvé dans la majorité des palmeraies de la région, mais avec une moindre représentation en aval, du fait de l'amoindrissement des ressources hydriques. Le type 2 est surtout concentré autour des palmeraies proches de la localité de Zagora, du fait de la présence d'une coopérative de transformation laitière. Le type 3 est caractéristique des élevages ovins avec une adhésion à l'ANOC, qui bénéficient des subventions prévues pour la sélection de la race D'man. Ces élevages sont dispersés dans toute la vallée du Drâa. Enfin, le type 4 est surtout localisé dans les confins aval de la vallée, du fait des limites en eau et de la salinité, qui imposent des déplacements d'animaux tels que les camelins et les caprins dans de vastes et maigres pâturages.

\section{Discussion et conclusion}

À l'instar d'autres agro-écosystèmes du Sud de la Méditerranée, la traditionnelle association cultures-élevage est un moyen de composer avec l'adversité (Alary et al., 2012). Mais elle est bouleversée par la rapidité des évolutions socioculturelles et économiques : scolarisation plus longue, montée en puissance des individualismes, aspirations à de meilleurs revenus que ceux de l'agriculture, etc. L'intensification et la spécialisation apparaissent comme un moyen de réaliser un projet capitaliste générateur de plus de revenus.
Cette étude démontre que pareille volonté concerne aussi l'élevage. Cela se traduit principalement par l'intensification de la production ovine, centrée autour de la sélection de la race D'man. Cette tendance implique la mobilisation de moyens financiers importants (constitution d'un noyau de reproducteurs, achats d'aliments, construction de bergeries aux normes, etc.), dans le but de bénéficier des subventions destinées à la sélection des futurs reproducteurs. Ce début de spécialisation transparaît clairement dans le type « élevage avec intensification de la production ovine » identifié par cette étude. À un degré moindre, il est aussi retrouvé dans le type « élevage basé sur l'espèce bovine ». Toutefois, ces stratégies comportent des risques, liés aux dysfonctionnements de la reproduction et à une maîtrise sanitaire insuffisante qui génère des taux de mortalité supérieurs aux normes établies. De plus, l'usage de la race D'man dans des programmes de croisement ovin pour l'amélioration de la prolificité risque de ne pas être adapté aux desiderata du marché au Maroc, qui est devenu fortement polarisé autour de la fête annuelle de l'Aïd Al Adha et ses exigences en termes d'apparence extérieure des animaux : cornage, conformation, etc. (Sraïri, 2011). Pour l'espèce bovine, l'étude révèle aussi des apports énergétiques limités en comparaison aux ovins (par UGB) qui handicapent ses performances, surtout dans des élevages utilisant des vaches de races importées. Il est d'ailleurs permis de questionner la pertinence du choix de bovins de races laitières dans pareil environnement, vu qu'elles n'affichent qu'une adaptation mitigée dans des régions plus favorables, au Nord du pays (Sraïri et Kiade, 2005). De plus, les difficultés d'écoulement du lait et de ses dérivés sur le marché local génèrent des tensions dans les unités industrielles de transformation, compromettant le paiement régulier et stable des éleveurs. Les contraintes de l'intensification agricole dans les oasis ne s'appliquent d'ailleurs pas exclusivement à l'élevage, puisque l'introduction récente de la culture de la pastèque s'est accompagnée de virulentes critiques, du fait de la pression qu'elle entraîne sur des ressources hydriques souterraines déjà 
surexploitées, et ce dans un contexte de désertification et d'ensablement (Benmohammadi et al., 2001). Ces velléités d'intensification de l'élevage soulèvent d'ailleurs un ensemble de questions, face à une incertitude climatique accrue et à la remise en cause de la durabilité de nombreuses activités agricoles (Chelleri et al., 2014).

L'étude confirme aussi les caractéristiques classiques de la polyculture-élevage dans les oasis, notamment la plurispécificité qui vise à limiter les risques en investissant différentes niches écologiques. Cela est pleinement illustré par le type « élevage plurispécifique ». Cela est aussi le cas pour le type «élevage à finalité pastorale », qui compte sur la plurispécificité d'animaux placés en système pastoral, voire nomade, avec la présence quasi exclusive des caprins et des dromadaires. De ce fait, les différents types d'élevage identifiés dans cette étude investissent l'espace oasien dans sa totalité, en valorisant ses ressources hydriques et édaphiques limitées. Toutefois, il est noté l'absence d'interrelations entre ces différents types, sans véritables échanges d'intrants ou de savoirs entre les divers opérateurs.

L'étude révèle aussi que le groupe familial, qui représentait un atout pour assumer les diverses tâches liées à l'élevage, constitue désormais une contrainte du fait de ses exigences pour une meilleure rémunération. Pareil paradoxe entre les obligatoires travaux d'astreinte de l'élevage et les revenus limités qui en sont tirés, et qui ne peuvent permettre à eux seuls à la population oasienne de s'extirper de la précarité, est retrouvé dans d'autres contextes (Sraïri et al., 2013). Face à cette situation, des sources alternatives de revenus sont recherchées (tourisme, industrie du cinéma, migration ; de Haas, 2006), mais cela peut entraver l'essor futur de l'élevage qui pourrait se voir privé d'une partie de sa main-d'œuvre.

L'autre constat prégnant de cette étude consiste en des performances zootechniques généralement limitées, aussi bien au plan de la production que de la reproduction. Cela est la résultante de plusieurs facteurs dont les plus significatifs sont l'inadéquation entre les rations distribuées aux animaux et leurs besoins effectifs, aussi bien en termes de quantité (matière sèche) qu'en termes de qualité (déséquilibres entre les apports protéiques et énergétiques, $\mathrm{du}$ fait de rations où le fourrage de base est la luzerne). En outre, l'absence de géniteur, surtout remarquée pour l'espèce bovine mais qui peut aussi être rencontrée chez les ovins, génère des retards de gestation et, par conséquent, la diminution de la production. Un décalage est ainsi remarqué entre les potentiels génétiques des races exploitées (surtout les ovins D'man), tels que les présentent les recherches en station (Kerfal et al., 2005), et les performances zootechniques observées chez les éleveurs. Les limites du milieu naturel (manque d'eau, stress thermique, surfaces exiguës, etc.) combinées à la méconnaissance des éleveurs des besoins de leurs animaux et de leur évolution le long de l'année, expliquent ces contreperformances. Finalement, toutes les exploitations évoquent le problème récurrent du manque d'encadrement et de formation professionnelle qui leur permettraient d'améliorer les performances de leur système de production. Ces constats renforcent le rôle possible des politiques publiques qui devraient viser en priorité l'amélioration des revenus des différentes catégories d'éleveurs identifiées, la valorisation des ressources naturelles des divers étages agro-écologiques de la région et le contrôle du territoire dans une zone frontalière. Pour ce faire, l'accompagnement des éleveurs dans des projets viables, prenant en compte les limites du contexte local (eau, foncier, pouvoir d'achat des populations, etc.), est crucial, afin d'éviter les désillusions du calquage de modèles ayant fait leurs preuves dans des milieux différents. C'est donc vers la prise en compte de la diversité des types d'élevages qu'il faudrait s'orienter, afin de consolider l'équité dans l'usage des moyens d'appui et d'enclencher un début d'intensification écologique à même de valoriser de manière optimale les ressources locales, tout en intégrant les sévères limites du milieu naturel.

Remerciements. Cette étude a été soutenue par la FAO, sous forme d'un appui financier à la conduite des enquêtes de terrain. Les auteurs voudraient aussi remercier les autorités agricoles locales (Office régional de mise en valeur agricole de Ouarzazate) pour leur soutien logistique. Une mention spéciale est réservée aux éleveurs qui ont accepté de répondre aux questions prévues dans les formulaires d'enquête et de suivi.

\section{Références}

Alary V, Daoud I, Abdelzaher M, Salama O, Aboul-Naga A, Merveille N, et al. 2012. Adaptation des sociétés bédouines de la côte Nord-Ouest de l'Égypte au changement global. Autrepart 62: 183-200.

Benmohammadi A, Benmohammadi L, Ballais JL, Riser J. 2001. Analyse des inter-relations anthropiques et naturelles : leur impact sur la recrudescence des phénomènes d'ensablement et de désertification au Sud-Est du Maroc (vallée de Drâa et de Ziz). Sécheresse 11: 297-308.

Bouix J, Kadiri M, Chari A, Ghanime R, Rami A. 1977. Fiche signalétique de la race D'man. Hommes, Terre et Eaux 25: 9-11. Disponible sur http://www.anafide.org/doc/HTE 25/25-2.pdf.

Chelleri L, Minucci G, Ruiz A, Karmaoui A. 2014. Responses to drought and desertification in the Moroccan Drâa valley region: resilience at the expense of sustainability? International Journal of Climate Change: Impacts \& Responses 5: 17-33.

de Haas H. 2006. Migration, remittances and regional development in Southern Morocco. Geoforum 37: 565-580.

Dollé V, Toutain G, Ferry M. 1989. Situation des systèmes oasiens en régions chaudes. Les Cahiers de la Recherche Développement 22: $3-14$.

Jouve P. 2012. Les oasis du Maghreb, des agro-écosystèmes de plus en plus menacés. Comment renforcer leur durabilité ? Courrier de l'Environnement de l'INRA 62: 113-121.

Karmaoui A, Messouli M, Khebiza MY, Ifaadassan I. 2014. Environmental vulnerability to climate change and anthropogenic impacts in dryland (pilot study: Middle Drâa Valley, South Morocco). Earth Science and Climate Change 5: 12 p.

Kerfal M, Chikhi A, Boulanouar B. 2005. Performances de reproduction et de croissance de la race D'Man au domaine expérimental de l'INRA d'Errachidia au Maroc. Rencontres Recherches Ruminants 12: 206. Disponible sur http://www. journees3r.fr/IMG/pdf/2005_systemes_10_kerfal.pdf.

Rignall K. 2015. The labor of agrodiversity in a Moroccan oasis. The Journal of Peasant Studies 43: 711-730. 
Ryschawy J, Choisis N, Choisis JP, Gibon A. 2013. Paths to last in mixed crop-livestock farming: lessons from an assessment of farm trajectories of change. Animal 7: 673-681.

Schilling J, Korbinian PF, Hertig E, Scheffran J. 2012. Climate change, vulnerability and adaptation in North Africa, with focus on Morocco. Agricultural Ecosystems and Environment 156: 12-26.

Sraïri MT. 2011. Le développement de l'élevage au Maroc : succès relatifs et dépendance alimentaire. Courrier de l'Environnement de l'INRA 60: 91-101.
Sraïri MT, Kiade N. 2005. Typology of dairy cattle farming systems in the Gharb irrigated perimeter, Morocco. Livestock Research for Rural Development 17: 1. Disponible sur http://www.cipav.org.co/ 1rrd//rrd17/1/sra171.htm.

Sraïri MT, Bahri S, Kuper M. 2013. Le travail et sa contribution aux stratégies d'adaptation de petites exploitations agricoles familiales mixtes d'élevage bovin/polyculture au Maroc. Biotechnologies, Agronomie, Science et Environnement 17: 463-474.

Citation de l'article : Sraïri MT, Azahra M'ghar F, Benidir M, Bengoumi M. 2017. Analyse typologique de la diversité et des performances de l'élevage oasien. Cah. Agric. 26: 15005. 